\title{
Job Scheduling based on Harmonization between the Requested and Available Processing Power in The Cloud Computing Environment
}

\author{
Elhossiny Ibrahim \\ Computer Science \& Eng. \\ Dept., Faculty of Electronic \\ Eng. Menouf 32952, Egypt
}

\author{
Nirmeen A. El- \\ Bahnasawy \\ Computer Science \& Eng. \\ Dept., Faculty of Electronic \\ Eng. Menouf 32952, Egypt
}

\author{
Fatma A. Omara \\ Faculty of Computers \& \\ information, Cairo University, \\ Egypt.
}

\begin{abstract}
The Cloud Computing is a most recent computing paradigm where IT services are provided and delivered over the Internet on demand and pay as you go. On the other hands, the task scheduling problem is considered one of the main challenges in the Cloud Computing environment, where a good mapping between the available resources and the users's tasks is needed to reduce the execution time of the users' tasks (i.e., reduce make-span), in the same time, increase the degree of capitalization from resources (i.e., increase resource utilization).

In this paper, a new task scheduling algorithm has been proposed and implemented to reduce the make-span, as well as, increase the resources utilization by considering independent tasks. The proposed algorithm is based on calculating the total processing power of the available resources (i.e., VMs) and the total requested processing power by the users' tasks, then allocating a group of users' tasks to each VM according to the ratio of its needed power corresponding to the total processing power of all VMs.

To evaluate the performance of the proposed algorithm, a comparative study has been done among the proposed algorithm, and the existed GA, and PSO algorithms. The experimental results show that the proposed algorithm outperforms other algorithms by reducing make-span and increasing the resources utilization.
\end{abstract}

\section{Keywords}

Cloud Computing; Task scheduling; Particle swarm optimization; Genetic Algorithm.

\section{INTRODUCTION}

According to the Cloud Computing, the computational resources and services of the data centers are allowed to be used by the users (i.e., machines, network, storage, operating systems, application development environments, application programs) through the Internet by using mobile, PC, laptop, PDA, etc...(see Fig 1) [1] [2]. On the other hand, the selfprovisioning of IT resources is considered one of the main features of the Cloud Computing [3]. The Cloud Computing deplyment types are public, private, hybrid, community, and federated, while the Cloud Computing services are categorized as Software as a Service (SaaS), Platform as a Services (PaaS), and Infrastructure as a Services (IaaS) [4]. Therefore, , the users can easily choose the suitable cloud services and types which fit their requirements $[1,3]$.

The main issues to be concerned in the Cloud Computing are the resource management and task scheduling. Therefore, the Cloud providers should provide the services, and deploy virtual machines (VMs), as well as, defin the scheduling policies to allocate VMs to the users' tasks [5].

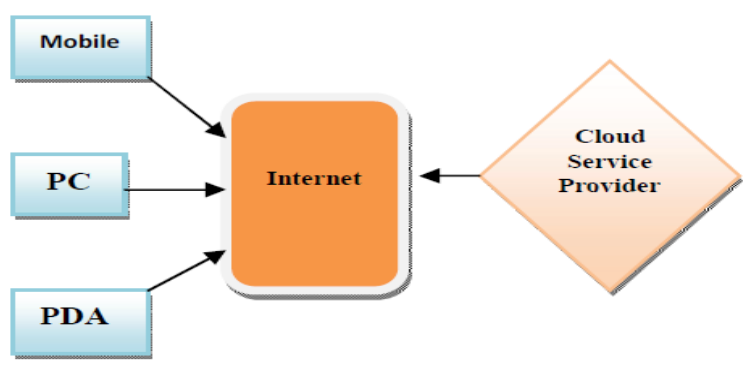

Fig 1: Cloud Computing Services Access.

In this paper, a new task scheduling algorithm has been proposed. The main principle of the proposed algorithm is based on calculating the total processing power of the available resources (i.e., VMs) and the total requested processing power by the users' requests, then allocating a group of the users's requests to each VM according to the ratio of its needed power corresponding to the total processing power of all VMs.To evaluate the performance of the proposed algorithm, a comparative study has been done among the proposed algorithm, the existed GA, and PSO algorithms. The experimental results show that the proposed algorithm outperforms other algorithms by minimizing the total execution time of the users' requests and increasing the utilization of the resources.

The remainder of the paper is organised as follows. In Section 2 , the related work is illustrated followed by the fundamental of the proposed task scheduling algorithm in Section 3. In Section 4, the performance evaluation of the proposed algorithm via Cloudsim simulator and the comparative study are discussed. Finally, in Section 5, conclude the contributions and point out the future work.

\section{RELATED WORK}

Yun Yang, Ke Liu, and Jinjun Chen [6] have proposed an innovative transaction intensive cost-constraint scheduling algorithm to minimize the cost and the execution time. The simulation results proved that this algorithm could minimize the cost while meeting the user designated deadline. Suraj Pandey et al. [7] have proposed a heuristic task scheduling to minimize the cost of task-resource mapping using particle swarm optimization (PSO) algorithm. According to the simulation results, it is found that the proposed algorithm can achieve as much as 3 times cost savings as compared to BRS, and good distribution of workload onto resources. Ke Liu et 
al. [8] have presented a Compromised-Time-Cost (CTC) scheduling algorithm. The CTC algorithm considers the characteristics of Cloud Computing to accommodate instanceintensive cost-constrained workflows by compromising the execution time and cost of the users' tasks which are enabled on the fly. The simulation results has demonstrated that the CTC algorithm can achieve lower cost while meeting the user-designated deadline or reducing the mean execution time within the user- designated execution cost. Saeed Parsa and Reza Entezari-Maleki [9] have proposed a task scheduling algorithm called Resource-Aware-Scheduling (RASA). It is considered an amalgamation of two traditional scheduling algorithms; Max-min and Min-min. Therefore, the deadline of each task, arriving rate of the tasks, cost of the task execution on each of the resource, and cost of the communication are not considered .The experimental results proved that RASA algorithm outperforms the existing scheduling algorithms in large scale distributed systems. J.Huang [10] has proposed a workflow task scheduling algorithm based on the genetic algorithms (GA) model in the Cloud Computing environment. The experimental results proved that the efficiency of resource allocation has been satisfies, and in the same time, minimized the completion time. Lei Zhang et al. [11] have proposed a PSO algorithm. This proposed algorithm is similar to the genetic algorithms (GA). The aim of this algorithm is to improves the efficiency of resource allocation and minimize the completion time simultaneously. It is noted that the performance of PSO usually spent shorter time to accomplish the various scheduling tasks and specifies better result comparing to the GA algorithm. Also, they have proved that the PSO algorithm can get better effect for a large scale optimization problem. Cui Lin, and Shiyong Lu [12] have proposed an Scalable Heterogeneous Earliest-Finish-Time Algorithm (SHEFT) workflow scheduling algorithm to schedule a workflow elastically on a Cloud Computing environment. The experimental results show that SHEFT is not only outperform several representative workflow scheduling algorithms in optimizing workflow execution time, but also enable resources to scale elastically at runtime. Visalakshi and Sivanandam [13] have presented Hybrid Particle Swarm Optimization (HPSO) method for solving the Task Assignment Problem (TAP) by dynamically schedule heterogeneous tasks on to heterogeneous processors in a distributed setup. The HPSO yields a better result. The experimental result of the HPSO algorithm has proved that the PSO outperforms the GA algorithm. S.Selvarani, and G.Sudha Sadhasivam [14] have proposed an improved cost-based scheduling algorithm for making efficient mapping of tasks to available resources in the cloud. This scheduling algorithm divides all user tasks depending on the priority of each task into three different lists. This scheduling algorithm measures both resource cost and computation performance. Also, it improves the computation / communication ratio. Yang et al. [15] have highlighted the issue of job scheduling in the Cloud Computing with considering hardware/software failure and recovery. They have proposed a Reinforcement Learning (RL) based algorithm that helps the scheduler to define scheduling decision with fault tolerable while maximizing utilities attained in the long term.

\section{THE PROPOSED ALGORITHM}

According to the work in this paper, a new task scheduling algorithm on the Cloud Computing environment has been proposed. The main idea of the proposed algorithm is that allocating the available VMs to the requested tasks according to the following steps:-
[1] Calculate the total available processing power of the available VMs (i.e., VM_MIPS).

[2] Calculate the total requested processing power by the users' tasks (TASKS_MIPs).

[3] Calculate the power factor (PF) of each available resource (VMi) using equation (1).

$$
\begin{gathered}
\text { PF of VMi }= \\
\frac{\text { Processing Power of VMi }}{\text { Total Processing Power of All VMs }} .
\end{gathered}
$$

[4] Calculate the allotment for each existed resource (VMi) as equation (2).

$\mathrm{VM}_{\mathrm{i}}$ allotment $=\mathrm{PF} *($ total requested processing power (TASKS_MIPs))

[5] Search the requested tasks to find a task or a group of tasks which need processing power equal to or less than the allotment of the VMi as calculated in step (4) by considering that the different between the selected tasks processing power and VM allotment to be minimum.

The pseudo code of the proposed algorithm is as follows:

Input:

Number $n$ of cloudlets (i.e., tasks).

Number $\mathrm{m}$ of VMs (i.e., resources).

Output:

Mapping Scheme for the requested tasks (cloudlets) on the available resources (VMs).

// calculate the total processing power of cloudlets

1 for $\mathrm{i}=1$ to $\mathrm{n}$ do

2 Define MIPS of the cloudlet

3 endfor

// calculate the total processing power of VMs.

4 for $\mathrm{i}=1$ to $\mathrm{m}$ do

5 Define MIPS of the VMs

// calculate the power factor of each VM, and the allotment of each VM

6 for $\mathrm{i}=1$ to $\mathrm{m}$ do

7 PF of $\mathrm{VM}_{\mathrm{i}}=$ processing power of $\mathrm{VM}_{\mathrm{i}} /$ total processing power of all VMS

$8 \quad \mathrm{VM}_{\mathrm{i}}$ allotment $=\mathrm{PF} *($ total requested processing power (TASKS_MIPs).

9 find the sum of $\operatorname{set}\left(\mathrm{VM}_{\mathrm{i}}\right.$ allotment $)$.

10 endfor

11 Select a set of cloudlets with the sum of their MIPs equal to/or less $\mathrm{VM}_{\mathrm{i}}$ allotment.

12 return the set.

\section{PERFORMANCE EVALUATION}

To evaluate the performance of the proposed algorithm, a comparative study has been done among the proposed 
algorithm and the GA, PSO, and First-Come-First-Serve (FCFS) algorithms by considering the Make-span.

\subsection{Experimental Environment}

The proposed task scheduling algorithm has been written by java programming language using eclipse program in Intel(R) Core(TM) 2 Duo CPU in $2.10 \mathrm{GHZ}$ of processor and $4.00 \mathrm{~GB}$ of RAM, through the CloudSim simulator. On the other hands, CloudSim is an extensible simulation toolkit that enables modeling and simulation of Cloud Computing systems and application provisioning environments. The CloudSim toolkit supports the Cloud s components such as data centers, virtual machines (VMs), and resource provisioning policies. On the other hand, the Cloudsim could implement generic application_provisioning techniques that can be extended easly with limited efforts [16].

According to the implementation using Cloudsim, the VMs are considered the cloud resources and Cloudlets as tasks/jobs. The make-span of different algorithms have been measured by considering varying the cloudlets while VMs are fixed, as well as, varying VMs.

Cloudlets were generated from a standard formatted workload of a High performance computing center called HPC2N in Sweden as a benchmark. According to this benchmark, Each row in the workload represents a cloudlet where the first column is the id of the cloudlet, the length of the cloudlet from the fourth column (the runtime value multiplied by the rating which is defined as 1 MI in CloudSim), and finally the number of the requested processing elements from the eighth column [17].

\subsection{Experimental Results}

The experimental results of the proposed algorithm, PSO, GA, and FCFS algorithms are presented in Figs 2, 3, and 4, using different VMs, as well as, different cloudlets (jobs/tasks). According to the experimental results in Fig 2 with considering $5 \mathrm{VMs}$, it is found that the performance of the proposed algorithm outperforms PSO, GA, and FCFS algorithms with respect to the total execution time (i.e., makespan) by $59.68 \%, 71.1 \%$, and $47.2 \%$ respectively. The overall performance of the proposed algorithm (i.e., average improvement) is $59.35 \%$ relative to the PSO, GA, and FCFS algorithms.

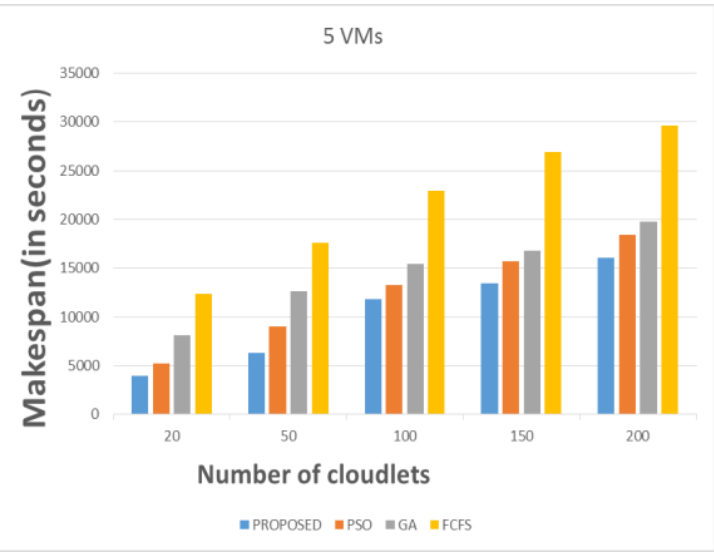

Fig 2: Make-span by considering 5 VMs

According to the experimental results in Fig 3 with considering $8 \mathrm{VMs}$, it is found that the performance of the proposed algorithm outperforms PSO, GA, and FCFS algorithms by $53.46 \%, 30.88 \%$, and $35.97 \%$ respectively. The overall performance of the proposed algorithm performance is $40.1 \%$ relative to the PSO, GA, and FCFS algorithms.

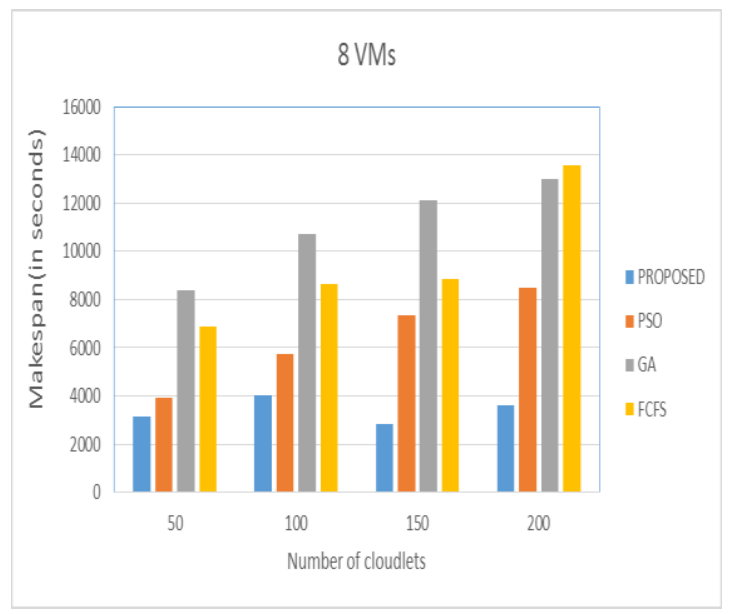

Fig 3: Make-span by considering $8 \mathrm{VMs}$

By considering $10 \mathrm{VMs}$, the experimental results in Fig 4 proved that the performance of the proposed algorithm outperforms PSO, GA, and FCFS algorithms by 55\%, 36\%, and $26.46 \%$ respectively. The overall performance of the proposed algorithm performance is $39.15 \%$ relative to the PSO, GA, and FCFS algorithms.

Generally, by calculating the average performance of the proposed algorithm, PSO, GA, and FCFS algorthms, it is found that the proposed algorithm outperforms PSO, GA, and FCFS by $57.7 \%, 51.2 \%$, and $39.89 \%$ respectively. And the overall performance of the proposed algorithm is $49.6 \%$ relative to PSO, GA, and FCFS algorithms.

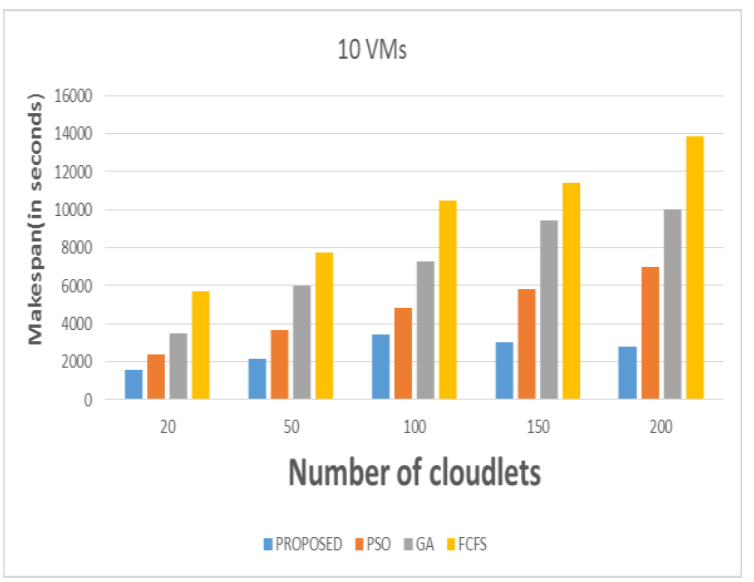

Fig 4: Make-span by considering $10 \mathrm{VMs}$

\section{CONCLUSION AND FUTURE WORK}

Task scheduling is one of the main issues in the Cloud Computing. Efficient task scheduling is essential for saving the time and utilizing the resources. In this paper, a task scheduling algorithm has been proposed for the Cloud Computing environment. The main idea of the proposed algorithm is that allocating the available VMs to the requested tasks by considering the processing power of VMs and tasks. To evaluate the performance of the proposed algorithm, a 
comparative study has been done among the proposed algorithm, PSO, GA, and FCFS algorithms. The experimental results prove the efficiency of the proposed algorithm by minimizing make-span by $49.6 \%$, in addition, increasing the utilization ratio. The proposed task scheduling algorithm could be further extended by considering the cost, as well as, the memory size. Also, the proposed algorithm could be modified by considering dependent tasks.

\section{REFERENCES}

[1] A. Soror, U. F. Minhas, A. Aboulnaga, K. Salem, P. Kokosielis, and S. Kamath, "Deploying Database Appliances in the Cloud.," IEEE Data Eng. Bull., vol. 32, No. 1, PP. 13-20, 2009.

[2] Rajveer Kaur, Supriya Kinger, "Analysis of Job Scheduling Algorithms in Cloud Computing", International Journal of Computer Trends and Technology (IJCTT), vol. 9 No. 7, PP. 379-386, Mar 2014.

[3] A.jangra, and T.Saini. "Scheduling Optimization in Cloud Computing." International Journal of Advanced Research in Computer Science and Software Engineering", IJARCSSE 3, PP. 62-65. April 2013.

[4] Handbook of Cloud Computing [online]. Available:http://www.springerlink.com/index/10.1007/9 78-1-4419-6524-0.

[5] Sanjaya K. Pandaa, Indrajeet Guptab and Prasanta K. Janac, " Allocation-

Aware Task Scheduling for Heterogeneous Multi-Cloud Systems", ScienceDirect, PP. 50176-184, 2015.

[6] Y. Yang, et al., " An Algorithm in SwinDeW-C for Scheduling Transaction- Intensive Cost-Constrained Cloud Workflows," Proc. of 4th IEEE International Conference on e-Science, Indianapolis, USA, PP. 374375, December 2008.

[7] Suraj Pandey, Linlin Wu, Siddeswara Guru, and Rajkumar Buyya. "A Particle Swarm Optimization (PSO)-based Heuristic for Scheduling Workflow Applications in Cloud Computing Environments." Proceedings of the 24th IEEE International Conference on Advanced Information Networking and Applications (AINA), Perth, Australia. April 20-23, 2010.
[8] Ke Liu, Hai Jin, Jinjun Chen, Xiao Liu, Dong Yuan, Yun Yang, " A Compromised-Time-Cost Scheduling Algorithm in SwinDeW-C for Instance-Intensive CostConstrained Workflows on a Cloud Computing Platform," International Journal of High Performance Computing Applications - IJHPCA, vol. 24, No. 4, PP. 445-456, 2010

[9] Saeed Parsa and Reza Entezari-Maleki," RASA: A New Task Scheduling Algorithm in Grid Environment" in World Applied Sciences Journal (Special Issue of Computer \& IT), PP. 152-160, 2009.

[10] J.Huang. "The Workflow Task Scheduling Algorithm Based on the GA Model in the Cloud Computing Environment." Journal of Software, vol. 9, No 4, PP. 873-880, April 2014.

[11] Lei Zhang, et al. "A Task Scheduling Algorithm Based on PSO for Grid Computing." International Journal of Computational Intelligence Research, vol. 4, No.1, PP. 37-43, 2008.

[12] Cui Lin, Shiyong Lu," Scheduling ScientificWorkflows Elastically for Cloud Computing" in IEEE 4th International Conference on Cloud Computing, 2011.

[13] Visalakshi, and Sivanandam. "Dynamic Task Scheduling with Load Balancing using Hybrid Particle Swarm Optimization." International Journal of Open Problems in Computer Science and Mathematics, ICSRS Publication, vol. 2, No. 3, PP. 476-488, 2009.

[14] Mrs.S.Selvarani1, Dr.G.Sudha Sadhasivam, "Improved Cost-based Algorithm for Task Scheduling in Cloud Computing, India, , IEEE , PP. 620-624, 2010.

[15] Yang, et al. "An Utility- based Job Scheduling Algorithm for Cloud Computing Considering Reliability Factor". Proceedings of the 2011 International Conference on Cloud and Service Computing, IEEE Xplore Press, Hong Kong, PP. 95-102, Dec. 12-14.

[16] S.Singh, M.Kalra, "Task Scheduling Optimization of Independent Tasks in Cloud Computing Using Enhanced Genetic Algorithm," International Journal of Application or Innovation in Engineering \& Management, vol. 3, Issue 7, PP. 2319 - 4847, July 2014.

[17] U. University, " The HPC2N Seth log, " 2006. [Online]. Available:http://goo.gl/wrxAK. 